\title{
Pattern of attempted suicide in Babylon in the last 6 years of sanctions against Iraq
}

\author{
Ali Abdurrahman Younis ${ }^{1}$ FRCPsych and Hamdy Fouad Moselhy ${ }^{2}$ MRCPsych
}

\author{
${ }^{1}$ Consultant Psychiatrist, Al Ain General Hospital, United Arab Emirates \\ ${ }^{2}$ Associate Professor of Psychiatry, Department of Psychiatry and Behaviors Sciences, Faculty of Medicine and Health Sciences, \\ UAE University, Al-Ain, United Arab Emirates, email hamdy.fouad@uaeu.ac.ae
}

$S^{u}$ uicide among Muslims and in Muslim countries is rare (Hocaoglu et al, 2007). Although much of the research has comprised simple descriptive studies, and despite the possible underreporting of suicidal behaviour in countries where such behaviour is illegal, suicide rates do appear to be lower among Muslims than among the followers of other religions, even in countries which have populations belonging to several religious groups (Lester, 2006). However, rates of attempted suicide do not appear to be lower in Muslims than in non-Muslims (Pritchard \& Amanullah, 2007), possibly because although there are strong religious sanctions against suicide, there are no clear principles regarding attempted suicide.

Iraqis (most of whom are Muslims) were generally subject to great worry regarding life domains such as finance, security and politics during the period of sanctions. Sanctions against Iraq were imposed by the United Nations on 6 August 1990, following the invasion of Kuwait in 1990, and they continued until the US-led invasion of Iraq in 2003. The economic sanctions resulted in high rates of malnutrition, lack of medical supplies and diseases from lack of clean water. They seriously hampered the Iraqi healthcare system in many ways: cutting imports of drugs and equipment; slowing resumption of local drug production; causing an exit of foreign medical and nursing staff; and restricting contacts between Iraqi doctors and outside experts.

In this study we aim to describe the pattern of attempted suicide in the Babylon governorate of Iraq during the last 6 years of sanctions.

\section{Method}

We conducted a retrospective case-note review, looking at attempted suicide over 6 years. The study population consisted of all persons referred to Merjan General Hospital, Al-Hilla City, in the Babylon governorate, between May 1996 and May 2002, following self-poisoning or self-injury. The hospital receives all referred cases from Al-Hilla City and the surrounding area. Patients who are believed to have attempted suicide are routinely referred from the accident and emergency department to the psychiatric service in the hospital. All patients so referred receive a detailed psychosocial assessment by the only psychiatrist (AAY). The psychiatric service in the hospital is the only one for the whole governorate. Merjan General Hospital has an open referral policy (i.e. it is free of charge) and has 400 beds. It serves the whole Babylon governorate (1 200000 people).
A specially designed data sheet was developed and a pilot analysis undertaken on 20 sets of medical notes. The data sheet was then revised and all the admissions taken on by the service in the study period were reviewed. Demographic data, descriptive psychopathology prior to presentation and mental state examination on assessment were recorded.

The review board of Merjan General Hospital approved the study.

\section{Statistical analysis}

Analyses were performed using the Statistical Package for Social Sciences (SPSS, version 14). Descriptive statistics were used to summarise the sociodemographic and clinical characteristics of the sample. A chi-square test was used to compare non-parametric data. All statistical tests were considered significant at $P=0.05$

\section{Results}

\section{Characteristics of participants}

In the 6-year study period, 90 individuals who had attempted suicide were seen at the accident and emergency department. Females outnumbered males by $4.6: 1$ (74:16). The mean age was 24 years (range 14-45 years) and most (77) (86\%) of all attempters were young adults aged 14-34 years. There were significantly more housewives than other categories of occupation $\left(59,66 \%, \chi^{2}=64.8, P=0.001\right)$; other categories were: employed $(6,7 \%)$, student $(14,16 \%)$ and unemployed $(11,12 \%)$. Most of the women were single $(34,38 \%)$; 21 were married (23\%), two divorced (2\%), one widowed (1\%) and the marital status was unkown for 32 (26\%). Thirty-eight came from an urban and 52 from a rural area.

Of all the attempts, 96\% (86 of 90) were by self-poisoning, 56\% (50) with organophosphates (usually pesticide), $40 \%$ (36) with drugs (34 paracetamol and 2 psychotropic medications); $48 \%$ (43) of the pesticide self-poisonings were by women. Only 4 females attempted suicide by self-immolation (4\%). Many suicide attempts were impulsive: of the 61 patients in the study sample treated as in-patients, 42 (69\%) reported considering suicide for less than 30 minutes before their attempts.

\section{Psychiatric diagnosis and prescribed psychotropic medications}

Of the 90 individuals, 73 (81\%) received a psychiatric diagnosis that met ICD-10 criteria. Fifty-eight (64\%) had an 
adjustment disorder, 12 (13\%) had post-traumatic stress disorder and $3(3 \%)$ a severe depressive disorder; none of the rest had syndromal psychopathology.

Only two patients had previously been involved with the psychiatric service. Both had a depressive disorder; one was on imipramine (125 mg daily) and the other on imipramine (175 mg daily) and haloperidol (10 mg daily). None of the other patients was on any prescribed psychotropic medication. There was no drug or alcohol use problem among the whole sample.

On admission to the hospital, consciousness was clinically impaired in 38 patients (42\%) and 23 patients (26\%) had lost consciousness.

There was a statistically significant peak in suicide attempts in the year 1997 (27 patients, 30\%, $\chi^{2}=30.6$, $P=0.001)$. Winter was the season with the most suicide attempts among males and spring among females $\left(\chi^{2}=6.9\right.$, $P=0.03)$; students also had a significantly higher rate in spring $\left(\chi^{2}=33.7, P=0.001\right)$.

The disposal of the patients after assessment was as follows: 8 patients (9\%) were referred to the psychiatric outpatient department, 21 (23\%) were admitted as psychiatric in-patients and 61 (68\%) continued to be looked after on the medical ward, but were seen in the psychiatric out-patient department at least once for follow-up.

\section{Discussion}

The study characterised suicide attempts in a small city in Iraq during the last half of the sanctions period, which proved the most difficult time. The study sample was marked by a high proportion of psychiatric diagnoses (81\%). However, only two patients had previously been treated by the mental health service. Reasons for psychiatric patients in our culture not accessing services include fear of stigma, the absence of a national mental health service and resort to traditional healers. Stress-related diagnoses were the most frequent ( $96 \%$ of all diagnoses). The study supports the substantial roles of impulsive behaviour (Phillips et al, 2002) and acute stressors (Staehr \& Munk-Andersen, 2006) in suicidal behaviour.

As in other countries, suicide attempts in the present study were more common among women than men (Prosser et al, 2007). However, the ratio of $4.6: 1$ here is higher than the international average ratio of around $2: 1$ (Schmidtke et al, 1996). This is possibly because, although war creates acute and long-lasting health problems in both men and women, many aspects of war affect the health of women disproportionately, through societal changes that may subordinate them and not prioritise their life and health (Arcel \& Kastrup, 2004). Furthermore, cultural norms affect what is acceptable behaviour and, despite the vital importance of women's work on farms, in factories and in civil defence, they were expected to retain their femininity. In Iraqi society, a failure to perform one's role as wife, mother or daughter may be interpreted as failure as a person (Kastrup, 2006).

Many similar studies internationally have reported high rates of substance use or intoxication with alcohol among people who attempt suicide. However, this was not the case in our sample, possibly for religious and social reasons.
Additionally, legislation at that time prohibited the drinking of alcohol in public places.

The limited availability of prescribed medications, including psychotropics, as a consequence of the economic sanctions would have reduced their use as a means of attempting suicide (only $2 \%$ ) compared with international studies, in which overdoses often predominate. Globally, legal restrictions on harmful organophosphates and herbicides have seen reductions in their use for attempted suicide, but in our sample a high proportion of suicide attempters ingested pesticide, possibly because more than half the sample came from rural areas (Merjan General Hospital is the receiving hospital for a predominantly agricultural hinterland) and the Iraqi government was supplying farmers with subsidised organophosphates. Self-immolation is a method of suicide generally used only by women from the Middle East (Al-Dabbas, 2006) and Central Asia (Campell \& Guiao, 2004); it has been reported in another Iraqi study (Carini et al, 2005). In our study it was limited to female patients (4\%) who suffered adjustment disorder. Little is known about this phenomenon and further research is needed.

The findings in this study are subject to at least three limitations. First, it was retrospective in design, and therefore vulnerable to bias related to missing data. Second, the nature of the work and the relatively small sample size make it difficult to generalise from these results. Finally, this retrospective study was limited to one hospital from a relatively small geographical district of Iraq, which may not be representative of the entire country.

\section{References}

Al-Dabbas, M. H. (2006) Deliberate self-burning: the psychosocial and clinical patterns among patients admitted to burn unit in King Hussein Medical Center, Jordan. Arabic Journal of Psychiatry, 12, 254-256.

Arcel, L. T. \& Kastrup, M. (2004) Women and health. Nordic Journal of Women's Studies, 12, 40-47.

Campbell, E. A. \& Guiao, I. Z. (2004) Muslim culture and female selfimmolation: implications for global women's health research and practice. Health Care for Women International, 25, 782-793.

Carini, L., Grippaudo, F. R. \& Bartolini, A. (2005) Epidemiology of burns at the Italian Red Cross hospital in Baghdad. Burns, 31, 687-691.

Hocaoglu, N., Kalkan, S., Akgun, A., et al (2007) A retrospective evaluation of analgesic exposures from Izmir, Turkey. Human Experimental Toxicology, 26, 629-636.

Kastrup, M. (2006) Mental health consequences of war: gender specific issues. World Psychiatry, 5, 33-34.

Lester, D. (2006) Suicide and Islam. Archives of Suicide Research, 10, 77-97.

Phillips, M. R., Li, X. \& Zhang, Y. (2002) Suicide rates in China, 19951999. Lancet, 359, 835-840.

Pritchard, C. \& Amanullah, S. (2007) An analysis of suicide and undetermined deaths in 17 predominantly Islamic countries contrasted with the UK. Psychological Medicine, 37, 421-430.

Prosser, J. M., Perrone, J. \& Pines, J. M. (2007) The epidemiology of intentional non-fatal self-harm poisoning in the United States: 20002004. Journal of Medical Toxicology, 3, 20-24.

Schmidtke, A., Bille Brahe, U., De Leo, D., et al (1996) Attempted suicide in Europe: rates, trends and sociodemographic characteristics of suicide attempters during the period 1989-1992. Results of the WHO/EURO Multicentre Study on Parasuicide. Acta Psychiatrica Scandinavica, 93, 327-338.

Staehr, M. A. \& Munk-Andersen, E. (2006) Suicide and suicidal behaviour among asylum seekers in Denmark during the period 2001-2003: a retrospective study. Ugeskrift for Laeger, 168, 1650-1653. 